\title{
A step-by-step guide to trans-axillary transcatheter aortic valve replacement
}

\author{
Morgan T. Harloff ${ }^{1}$, Edward D. Percy ${ }^{1}$, Sameer A. Hirji ${ }^{1}$, Farhang Yazdchi $^{1}$, Hunbo Shim ${ }^{1}$, \\ Muntasir Chowdhury ${ }^{1}$, Alexandra A. Malarczyk ${ }^{1}$, Piotr S. Sobieszczyk ${ }^{2}$, Ashraf A. Sabe ${ }^{1}$, Pinak B. Shah ${ }^{2}$, \\ Tsuyoshi Kaneko ${ }^{1}$
}

${ }^{1}$ Division of Cardiac Surgery, Brigham and Women's Hospital, Harvard Medical School, Boston, MA, USA; ${ }^{2}$ Division of Cardiovascular Medicine, Brigham and Women's Hospital, Boston, MA, USA

Correspondence to: Tsuyoshi Kaneko, MD. Division of Cardiac Surgery, Brigham and Women's Hospital, 15 Francis Street, Boston, MA 02115, USA.

Email: tkaneko2@partners.org.

\begin{abstract}
The application of transcatheter aortic valve replacement (TAVR) has expanded rapidly over the last decade as a less invasive option for the treatment of severe aortic stenosis. In order to perform successful TAVR, vascular access must be obtained with a large-bore catheter to deliver the transcatheter valve to the aortic annulus. Several techniques have been developed for this purpose including transfemoral (TF), trans-aortic, trans-apical, trans-caval, trans-carotid, and trans-axillary (TAx) with varying degrees of success. Among them, $\mathrm{TF}$ access is the most common and preferred method owing to its superior and well-established outcomes. However, in the setting of diseased iliofemoral arterial vessels, severe tortuosity, or iliofemoral arteries of insufficient caliber, TF access may not be possible. In these scenarios, one of the aforementioned alternative access routes needs to be considered. TAx-TAVR is an attractive alternative because it can be accomplished via access to a peripheral vessel as opposed to needing to enter the pericardial space or thoracic cavity. In addition, the open surgical cut-down procedure used to expose the axillary artery is familiar to cardiac surgeons who are accustomed to cannulating it for cardiopulmonary bypass. With advancements in TAVR technology including the evolution of delivery systems and corresponding smaller sheath sizes, total percutaneous access via the axillary artery is gaining substantial attention. In this review, we outline key aspects of patient selection, imaging and procedural techniques, and examine contemporary clinical outcomes with this approach.
\end{abstract}

Keywords: Transcatheter aortic valve replacement (TAVR); alternative access; trans-axillary (TAx) TAVR; Tax-TAVR

Submitted Apr 30, 2020. Accepted for publication Aug 25, 2020.
doi: 10.21037/acs-2020-av-79
View this article at: http://dx.doi.org/10.21037/acs-2020-av-79

\section{Introduction}

The commercial introduction of transcatheter aortic valve replacement (TAVR) in the United States in 2011 ushered in a paradigm shift in the management of severe aortic stenosis (1). Although initially limited in its infancy to patients who were at extreme or high-risk for surgical aortic valve replacement (SAVR), the PARTNER 3 and Evolut Low-Risk randomized trials in 2019 demonstrated non-inferiority of TAVR compared to SAVR in low surgical risk patients $(2,3)$. Shortly thereafter, the United States Food and Drug Administration (FDA) expanded approval for TAVR to include patients at all surgical risk profiles (4). Based on recent data from the Society of Thoracic Surgeons/American College of Cardiology Transcatheter Valve Therapy Registry, TAVR volumes have now surpassed SAVR volumes (5).

The transfemoral (TF) approach has become the most common and preferred vascular access route for TAVR as it is associated with the lowest complication rates compared to 
other alternative access techniques (6). However, anatomical limitations such as inadequate vessel caliber, extreme tortuosity, or extensive preexisting aorto-iliac atherosclerosis can render TF-TAVR challenging or impossible (7). When these conditions are present, alternative access sites should be carefully considered. These alternatives include transapical, direct-aortic, transcaval, trans-carotid, and transaxillary (TAx) TAVR. Trans-apical and direct-aortic TAVR were initially preferred, but have since fallen out of favor due to inferior outcomes as illustrated in the ad-hoc analysis of the randomized control studies comparing TAVR to SAVR in high-risk surgical patients $(8,9)$.

In contrast, TAx-TAVR has shown superior outcomes compared to trans-apical and aortic approaches with balloon-expandable valves, and similar outcomes compared to TF-TAVR with self-expanding valves $(6,7)$. In addition, the open surgical cut-down procedure used to expose the axillary artery is familiar to cardiac surgeons accustomed to cannulating it for cardiopulmonary bypass. Another advantage of TAx-TAVR compared to other alternative access sites is that it allows for a fully percutaneous approach with contemporary delivery systems. For these reasons, TAx-TAVR has developed into the second most common access route for TAVR when advanced iliofemoral disease renders TF access infeasible (6).

Herein, we discuss important aspects of TAx-TAVR within the framework of the following sequential steps: (I) patient selection; (II) imaging; (III) preparation; (IV) vascular access; (V) axillary access; (VI) valve deployment; (VII) closure.

\section{Procedural techniques}

\section{Patient selection}

Proper patient selection is critical to conducting successful TAx-TAVR. The heart team should consider this strategy for patients with symptomatic, severe aortic stenosis and anatomical limitations that render TF-TAVR access challenging. To be considered for TAx-TAVR, the crosssectional diameter of the subclavian and axillary arteries must be of sufficient diameter. Of the current commercially available devices, the CoreValve Evolut Pro+ System (Medtronic, Minneapolis, Minnesota) requires a minimum vessel diameter of $5.0 \mathrm{~mm}$ for the $23-29 \mathrm{~mm}$ sized valves and the Edwards SAPIEN 3 Transcatheter Heart Valve System (Edwards LifeSciences Corp, Irvine, California) requires a minimum axillary artery diameter of $5.5 \mathrm{~mm}$ for valve sizes $20-26 \mathrm{~mm}$, and $6.0 \mathrm{~mm}$ for $29 \mathrm{~mm}$ sized valves. Even in patients with iliofemoral disease, the axillary artery minimal luminal diameter is usually $>5.0 \mathrm{~mm}$, making both systems viable options (10). Both the left and right axillary can be used, but the left is preferred as it allows better alignment with the native annulus and less risk of jeopardizing carotid perfusion, which may be a concern when the right axillary artery is used (6). The selected vessel must be free of severe calcification, excessive tortuosity, steep angulation of the subclavian artery at the takeoff from the aortic arch ( $>80$ degrees), and significant root angulation ( $<70$ degrees for the left subclavian artery and $<30$ degrees for the right) (11). In patients with a history of coronary artery bypass graft and a patent left internal mammary artery (LIMA), TAx-TAVR can safely be performed if the diameter of the axillary artery at the takeoff of the LIMA is greater than $5 \mathrm{~mm}$ or $5.5 \mathrm{~mm}$. Percutaneous access is contraindicated in morbidly obese patients when the skin to axillary artery depth is greater than $10 \mathrm{~cm}$. The absolute and relative contraindications for TAx-TAVR are summarized in Table 1.

\section{Imaging}

Standard preoperative evaluation for TAx-TAVR includes a TAVR protocol computed tomography (CT). Preoperative TAVR-CT protocol of the chest, abdomen, and pelvis permits interrogation of the aortic root anatomy, coronary heights, widths of the sinuses of Valsalva, and absence of severe calcification or extreme tortuosity involving the axillary arteries (12). It also allows for accurate measurements of the axillary artery diameter.

\section{Preparation}

The patient is brought to the catheterization laboratory or hybrid operating room and placed in the supine position. A cardiac anesthesiologist, stationed at the head of the patient's bed, induces either conscious sedation for percutaneous or general anesthesia for a cut-down approach. Intravenous antibiotics are administered for surgical antimicrobial prophylaxis. The patient's arms are positioned at their side so that radial access, if needed, and axillary access can be obtained without having to mobilize the arms. The patient is prepped and draped per standard surgical convention. A sterile table extending perpendicularly away from the bed is placed next to the patient's shoulder on the side where 
Table 1 Contraindications for trans-axillary transcatheter aortic valve replacement

Absolute contraindications

- Inadequate vessel size (subclavian or axillary artery diameter $\leq 5 \mathrm{~mm}$ for self-expanding and $\leq 5.5 \mathrm{~mm}$ for balloon-expandable valves)

- Severe vessel calcification

- Excessive tortuosity of the subclavian or axillary artery

- Preexisting vascular injury (i.e., dissection)

- Steep subclavian to arch angulation (>80 degrees)

- Significant calcification involving the aortic arch

- Significant aortic root angulation ( $<70$ degrees for the left subclavian artery and $<30$ degrees for the right)

Relative contraindications

- Patent ipsilateral internal thoracic artery graft

- Ipsilateral A/V fistula

- Morbid obesity (soft tissue depth of $10 \mathrm{~cm}$ from skin to axillary artery for percutaneous access)

- Presence of ipsilateral permanent pacemaker

axillary artery access is to be established (Figure 1). The fluoroscopic monitors should be placed above the sterile table and the fluoroscopic camera itself placed on the side opposite the intended access site. This arrangement allows the providers to work separately during the portion of the case where femoral and axillary access are established or in tandem side-by-side during valve deployment.

\section{Vascular access}

Femoral arterial access is obtained by accessing the common femoral artery with a 7Fr sheath under ultrasound (US) guidance via the Seldinger technique. The common femoral vein is likewise accessed via the Seldinger technique with US guidance. If there is going to be consideration for use of a Sentinel Cerebral Protection System (Boston Scientific Corp., Marlborough, MA, USA), right radial artery access should also be obtained. Following the administration of systemic heparin with a goal ACT of 250-350, the established access points can safely be utilized for catheter delivery.

A 6Fr pigtail catheter is then advanced from the femoral artery sheath to the base of the coronary sinus. A root aortogram is obtained using LAO cranial angulation. A temporary transvenous pacemaker is floated from the femoral venous sheath and anchored into the trabeculae of the right ventricle. If complete percutaneous access is to be utilized, a 5Fr Impulse diagnostic catheter (Boston Scientific
Corp., Marlborough, MA, USA) should be introduced into the femoral sheath to access the subclavian artery on the side to be used for large bore sheath access. Through this catheter, a 0.018 " guidewire should be advanced into the subclavian artery and then into the brachial artery. This wire serves as a useful marker on ultrasound during axillary artery access (Figure 2). The wire is also utilized at the end of the procedure as a guide for balloons or stents if the axillary artery requires repair after sheath removal. Once the wire is in place, the IM catheter can be removed leaving the wire outside of the sheath. Through the sheath, a pigtail catheter can then be advanced to the aortic root. If utilized, the cerebral protection device can be advanced from the right radial sheath at this time into the aortic arch and deployed to cover the brachiocephalic and right common carotid arteries.

\section{Axillary access}

For open access, the left axillary artery can be exposed through a small infraclavicular incision in the deltopectoral groove. The pectoral muscle is separated along the muscle fibers and the pectoralis minor muscle is partially divided. Although the axillary artery lies in close proximity to the brachial plexus, nerve damage can be avoided with meticulous surgical technique while dissecting down to the axillary artery. The axillary artery is exposed, and control of the vessel is established by encircling the axillary artery with 
A

Operator 1

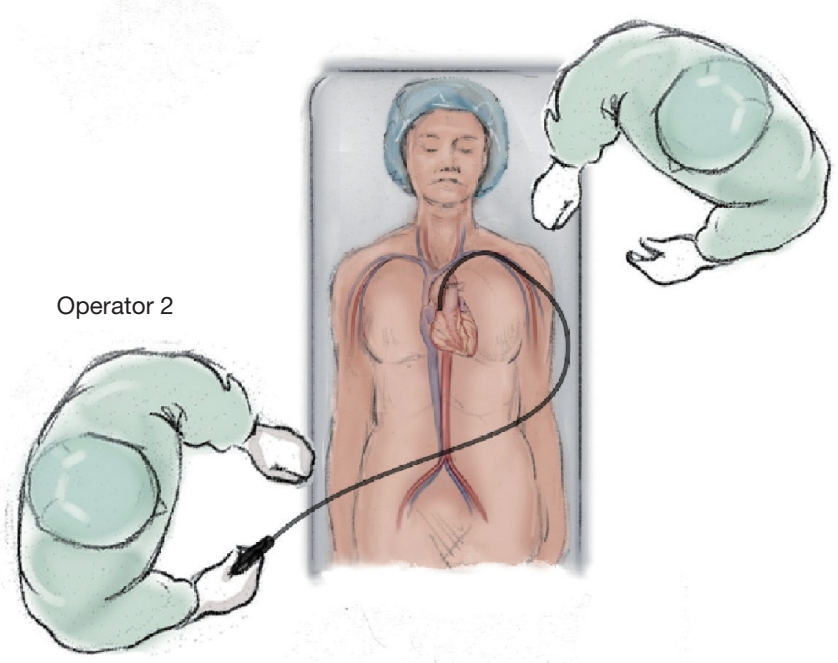

B

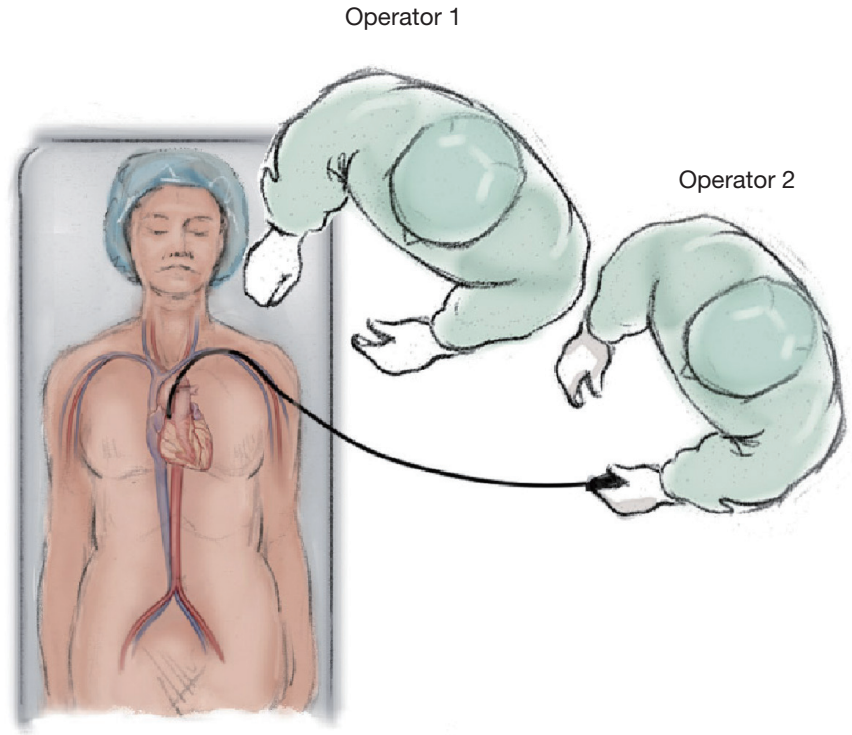

Figure 1 Room setup portraying the patient lying in supine position with their arms at their side. A sterile table extending perpendicularly away from the bed is placed next to the patient's shoulder on the side where axillary artery access is to be established. The fluoroscopic monitors should be placed above the sterile table and the fluoroscopic camera itself placed on the side opposite the intended access site. The providers can work separately during the portion of the case where femoral and axillary access are established (Option A) or in tandem (Option B) such as during device deployment.

soft rubber vessel loops proximally and distally (Figure 3).

The Seldinger technique is used with insertion of a $6 \mathrm{Fr}$ sheath between the vessel loops to access the axillary artery (Figure 4). A Bernstein catheter (Merit Medical, Rockland, MA, USA) is then inserted, and the valve is crossed. A Safari wire (Boston Scientific Corp., Marlborough, MA, USA) or
Lunderquist extra-stiff wire (Cook Group, Bloomington, IN, USA) is positioned within the left ventricular apex. Next, a large-bore sheath is inserted into the axillary artery (Figure 5). The valve is crossed, followed by the insertion of a large sheath, which is an important difference compared to the TF approach. It is important to watch the insertion 


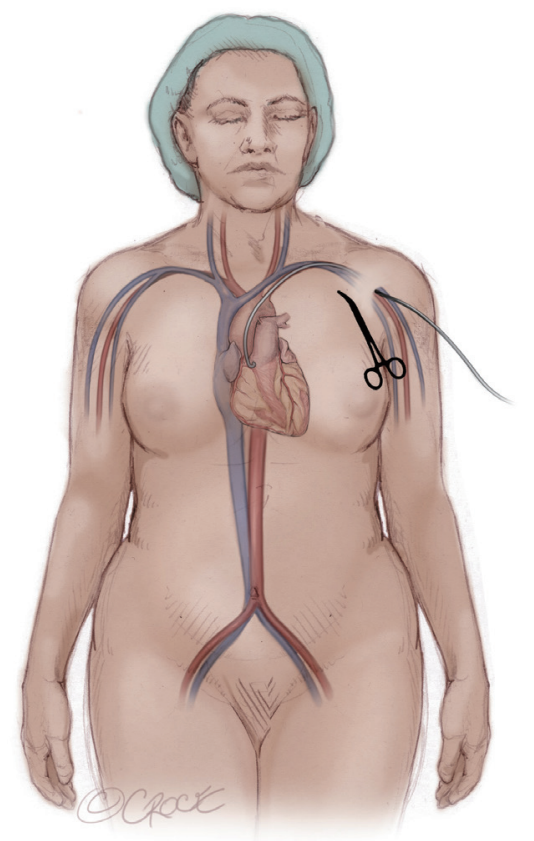

Figure 2 A guidewire is advanced into the left axillary artery via the right common femoral artery sheath to identify the vessel. A metallic instrument can be placed on the skin to outline the course of the artery in relation to the guidewire.

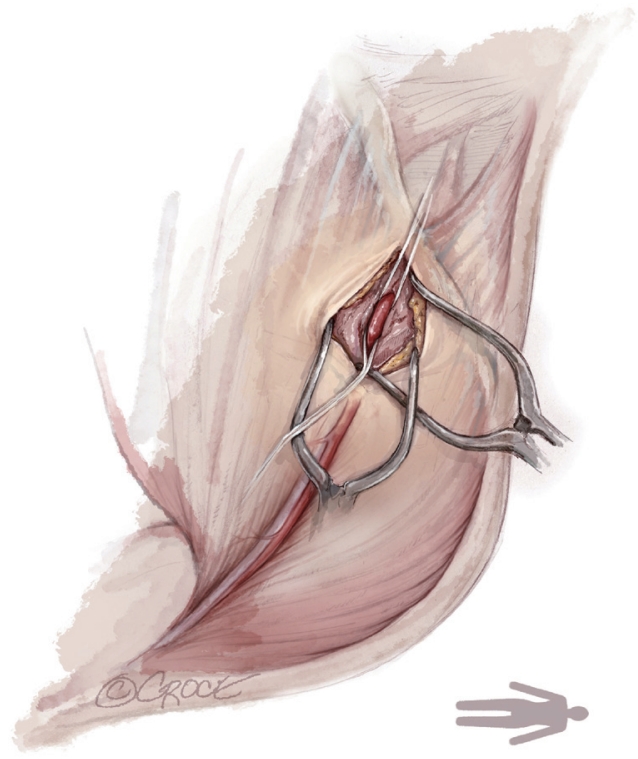

Figure 3 A small incision is made inferior to the clavicle in the deltopectoral groove. The dissection is carried down to the axillary artery. Control of the vessel is established by encircling the axillary artery with soft rubber vessel loops proximally and distally.

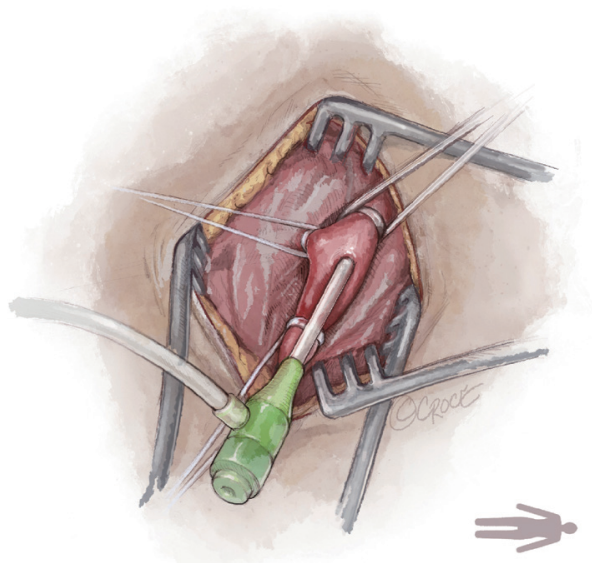

Figure 4 The Seldinger technique is used to insert a $6 \mathrm{Fr}$ sheath between the vessel loops to access the axillary artery.

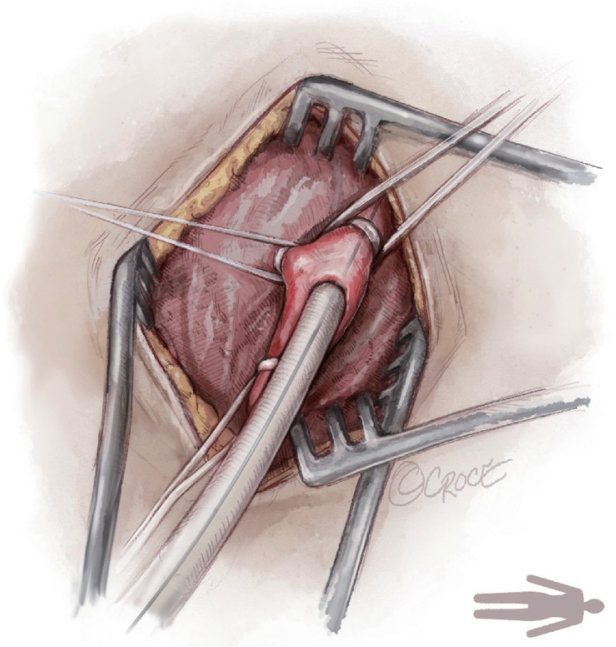

Figure 5 A large-bore sheath is inserted into the axillary artery over a stiff wire.

of the sheath under fluoroscopy since tortuosity of the vessel can create a kink in the sheath.

For complete percutaneous access, the inferior border of the clavicle and the medial edge of the pectoralis major muscle, near the deltopectoral grove, serve as superficial landmarks to identify the location of the axillary artery. A metallic instrument can be placed on the skin to outline the course of the artery in relation to the guidewire by fluoroscopy. Local anesthesia is administered to the soft tissue surrounding the axillary artery. Next, the left axillary 

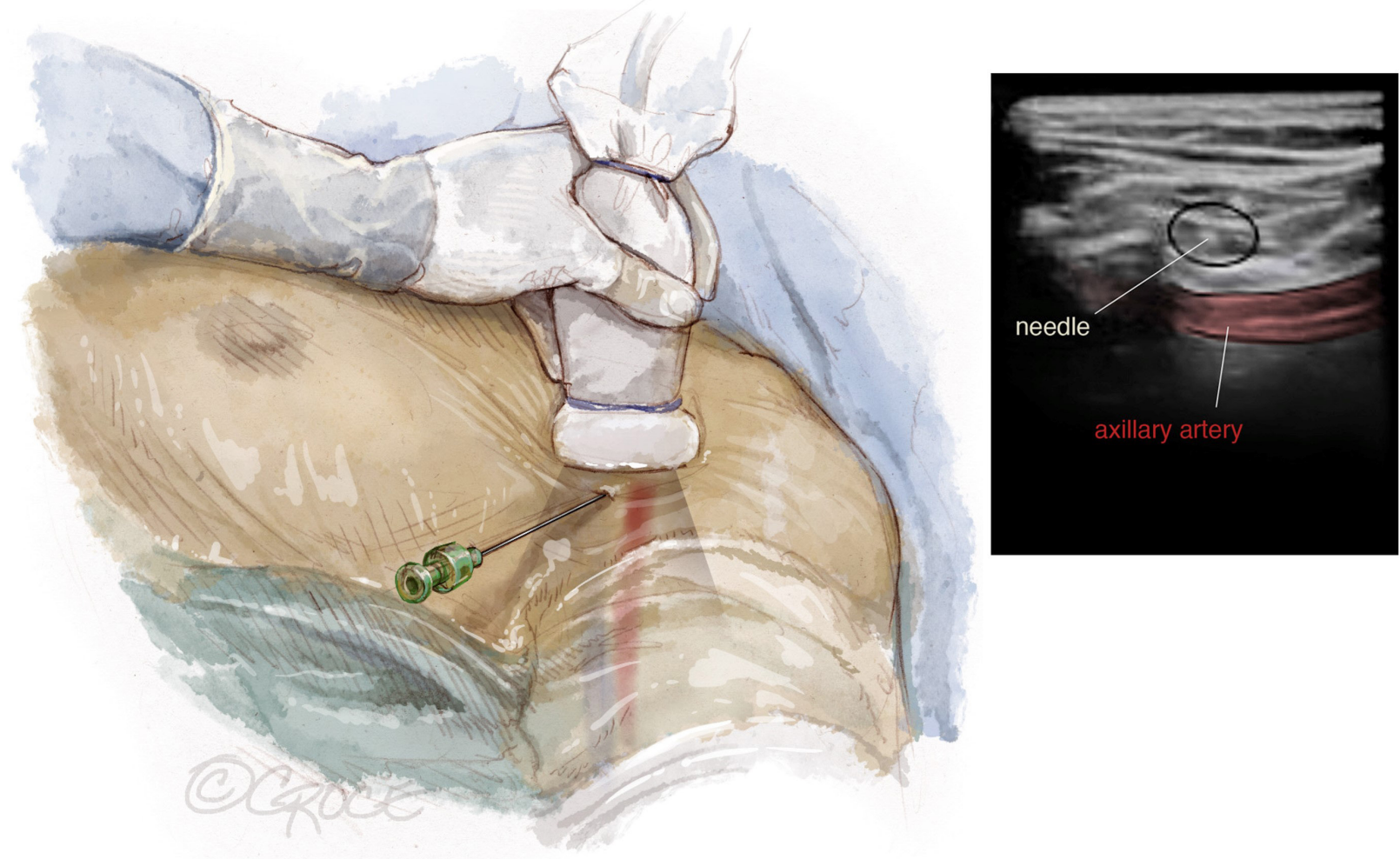

Figure 6 The axillary artery is punctured with a micro puncture needle under US and fluoroscopic guidance. An ultrasound image on the right demonstrates the tip of the needle (circle) heading toward the axillary artery (red) with the wire in it.

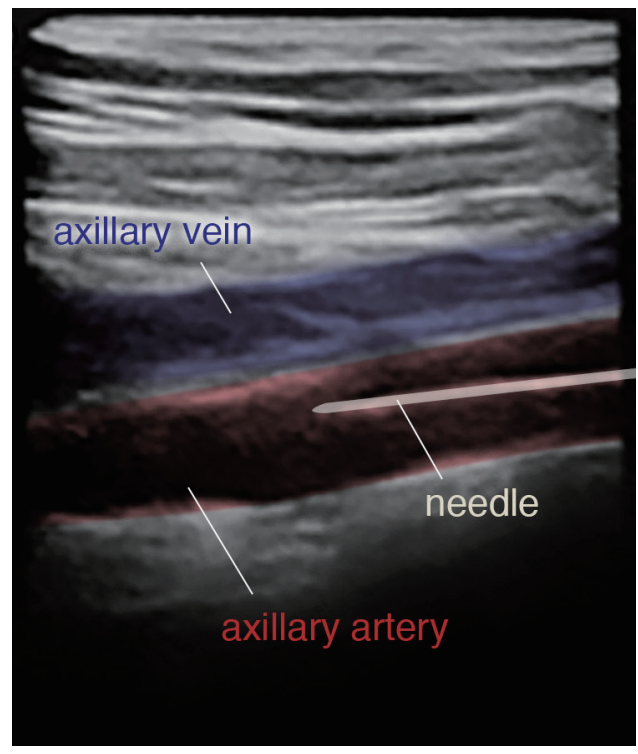

Figure 7 Ultrasound is used to visualize the 0.018 -inch wire in the axillary artery, distinguishing the artery from the axillary vein. artery is punctured with a micro puncture needle under US and fluoroscopic guidance (Figure 6).

The 0.018 -inch wire is visualized in the axillary artery, distinguishing the artery from the axillary vein (Figure 7). The angle of needle insertion should be as parallel to the skin as possible to minimize the likelihood of sheath kinking in the soft tissue. Additionally, the needle trajectory should be in the course of the axillary artery to avoid extra tension in the artery. The thoracoacromial branch is used as a marker for arterial puncture. The artery is punctured using a micropuncture system and once the wire is entered into the axillary artery, proper positioning is confirmed under fluoroscopy. The inner cannula of the micropuncture dilator can be advanced into the vessel and an angiogram can be taken to ensure appropriate positioning (Figure 8). The micropuncture wire is then re-introduced and the dilator upsized to accept a 0.035 -inch guidewire.

Subsequently, two Perclose Proglide (Abbott Laboratories, Chicago, IL, USA) devices are preemptively 




Figure $8 \mathrm{~A}$ micro puncture wire is advanced into the axillary artery. Fluoroscopy is used concurrently to document the wire advancing retrograde down the subclavian artery toward the aorta.

placed in the axillary artery for suture-mediated closure of the artery. Following the insertion of an $8 \mathrm{Fr}$ sheath, the native aortic valve is then crossed. The ensuing steps are identical to the open approach.

\section{Valve deployment}

Both the self-expanding CoreValve Evolut Pro+ System (Medtronic, Minneapolis, MN, USA) and the balloonexpandable Edwards SAPIEN 3 Transcatheter Heart Valve System (Edwards LifeSciences Corp, Irvine, California) can be used for Tx-TAVR. For the Sapien 3 valve, the device is advanced into the ascending aorta. The sheath is then pulled back into the axillary artery before the valve is pulled back to load into the balloon. For the Evolut system, the initially placed 14Fr sheath is removed, and the device is advanced into the axillary artery under fluoroscopic guidance. The remainder of the steps are similar to the TF approach. After confirming valve positioning with an aortogram (Figure 9), the valve is deployed under ventricular pacing (Figure 10). For a balloon-expandable valve, the heart is paced at 180 beats per minute in order to reduce forward flow during valve deployment. For a self-expanding valve, the heart need only be paced at $100-120$ beats per minute to stabilize the valve. Device position, expansion, paravalvular leak (PVL), and coronary perfusion are assessed using a combination of fluoroscopy and echocardiogram. After confirming satisfactory valve deployment, the delivery system is withdrawn from the left ventricle followed by catheter and sheath removal.

\section{Closure}

The delivery system and sheaths are removed from the axillary artery. For open access, the axillary artery is clamped both proximally and distally and repaired using 


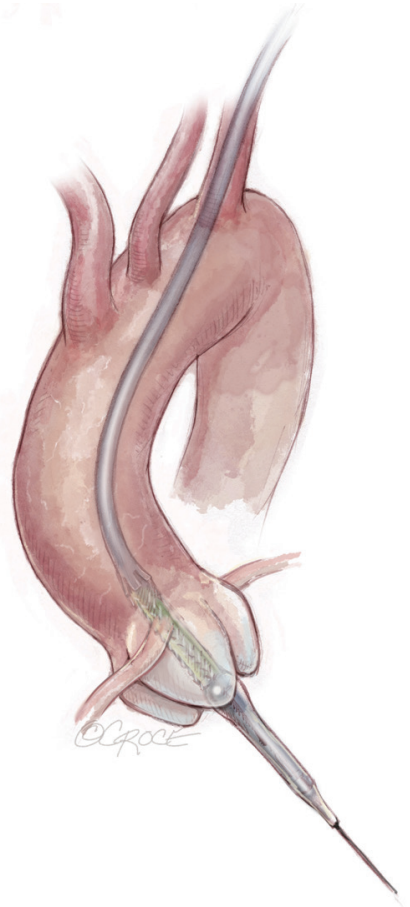

Figure 9 The delivery system is loaded over the stiff wire and carefully advanced in retrograde fashion across the aortic arch via the axillary artery and positioned within the annulus of the native aortic valve.

interrupted 5-0 Prolene sutures. Protamine is given following confirmation of intact repair. Care is taken to manage and tack all layers in case the artery is dissected. Endarterectomy may be needed in severe cases.

For percutaneous access, an appropriately sized peripheral balloon is advanced into the left axillary artery via the right femoral artery sheath and inflated to as low a pressure as possible (1-4 atmospheres) at the percutaneous insertion site to allow dry closure. This maneuver is performed to aid hemostasis and to ensure vascular control in the event of an access complication. Protamine is instituted and the Perclose Proglide devices are tied down over a wire at the insertion site to maintain vessel access. The wire is removed, and manual pressure is applied against the second rib. A completion angiogram is performed to ensure hemostasis, rule out vascular injury, and confirm patency of the left axillary artery (Figure 11). In case of residual bleeding, the vessel can be compressed against the thoracic cage to obtain hemostasis or a balloon can be used to tamponade the vessel.

Next, the femoral artery sheath is removed and the
Perclose device is used for closure. The femoral venous sheath is removed, and manual pressure is applied to achieve hemostasis. Finally, the cerebral protection device, if used, is removed along with the radial artery sheath. A TR band (Terumo Interventional Systems, Somerset, NJ) is placed on the wrist to obtain hemostasis at the radial artery access site.

\section{Comments}

\section{Clinical results}

The TAx approach is a rapidly growing alternative for TAVR when advanced peripheral arterial disease precludes the common femoral artery as a suitable access site. According to a recent TVT Registry report by McCabe et al., a total of 63,581 patients underwent TAVR across the United States with the SAPIEN 3 device between June 2015 and February 2018 (6). Of those, 3,628 (5.7\%) patients underwent non-TF TAVR (6). TAx-TAVR accounted for 1,249 (34.4\%) of those alternative access cases. The major vascular complication rate for TAx-TAVR was $2.5 \%$. When compared to traditional alternative access routes (trans-apical and trans-aortic), TAx-TAVR had lower 30 -day mortality rates $(5.3 \%$ vs. $8.4 \%, \mathrm{P}<0.01)$ as well as shorter intensive care unit and hospital lengths of stay, and lower incidence of new onset atrial fibrillation (Table 2). The stroke rate, however, was higher for the TAx group compared to trans-apical and trans-aortic $(6.3 \%$ vs. $3.1 \%$; $\mathrm{P}<0.05)$. For this reason, our preference is to utilize the left axillary approach, reserving the right side for use of a Sentinel embolic protection device.

Similar outcomes have been achieved with self-expanding delivery systems. For example, Gleason $e t$ al. demonstrated equivalent morbidity and mortality rates with TAx-TAVR compared to TF-TAVR using the Medtronic CoreValve (3). Clinical event rates including 30-day mortality, stroke, major bleeding, and major vascular complications were similar between the two groups (Table 3) (3). These encouraging results suggest that TAx-TAVR should be the preferred secondary access site for TAVR as it offers comparable outcomes to TF-TAVR.

A meta-analysis conducted by Amat-Santos et al. investigated whether or not TAx-TAVR provides similar outcomes to TF-TAVR (13). They found no difference in 30 -day mortality $(4.2 \%$ vs. $5.2 \%, \mathrm{P}=0.20)$, stroke $(3.7 \%$ vs. $3 \%, \mathrm{P}=0.15)$, or major vascular complications $(6.5 \%$ vs. $5.2 \%, \mathrm{P}=0.36)$ between TAx and TF respectively (Table 4) (13). Their results suggest that not only is TAx- 


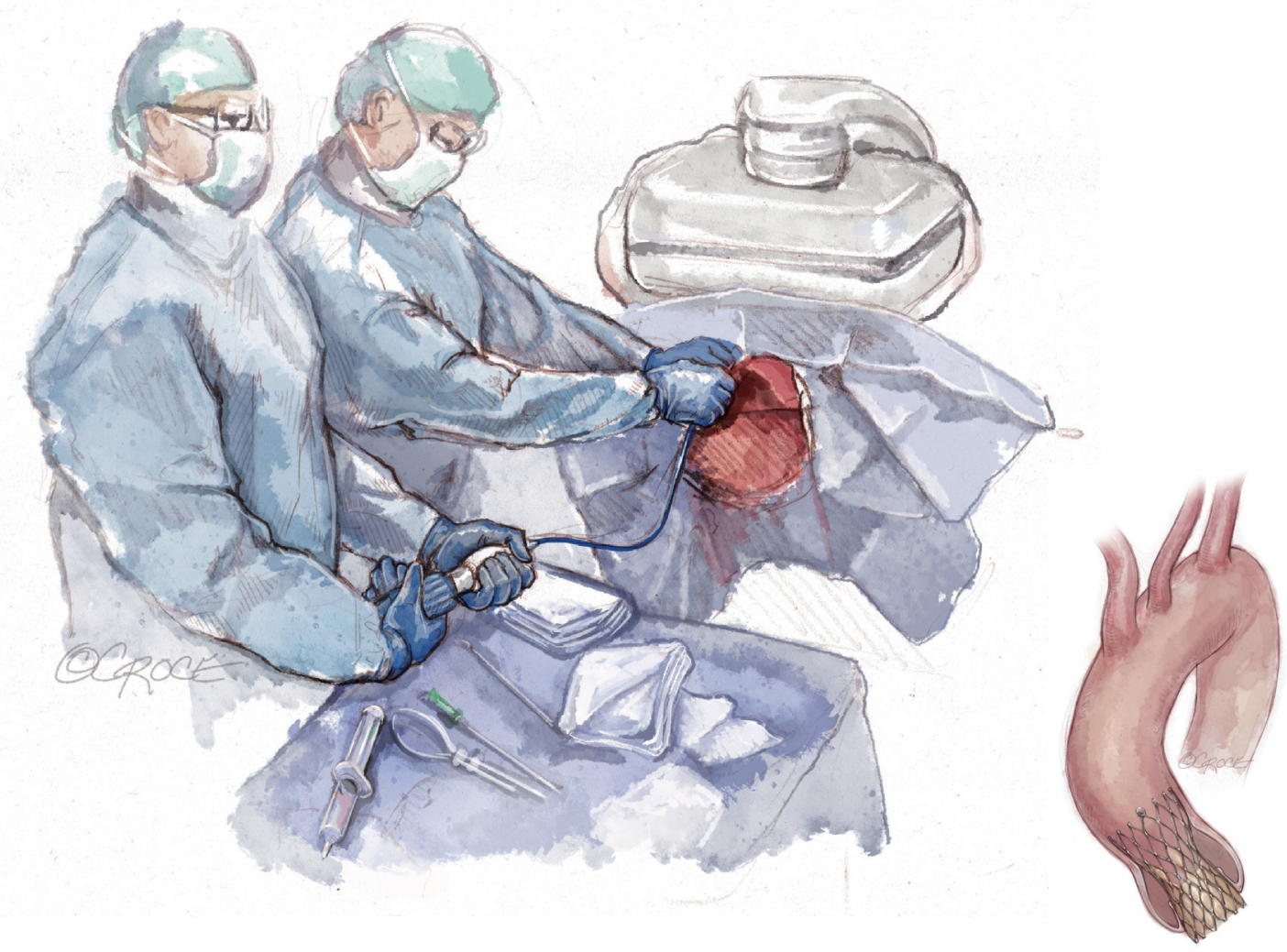

Figure 10 The valve is deployed under fluoroscopy with under ventricular pacing.

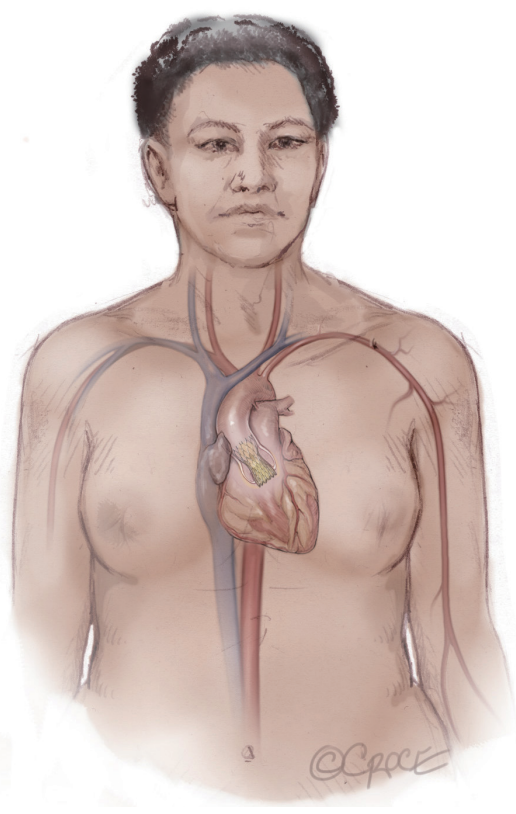

Figure 11 Completion angiogram is performed to ensure hemostasis, rule out vascular injury, and confirm patency the left axillary artery.
TAVR a suitable alternative to TF-TAVR, it may even be the preferred choice (13).

\section{Advantages and disadvantages}

The primary benefit associated with TAx-TAVR is that it avoids a thoracic incision and eliminates the need for entry into the pericardial space or thoracic cavity. The axillary cut down technique is familiar to cardiac surgeons since it is commonly used as a cannulation site for open heart surgery. Moreover, the percutaneous TAx-TAVR access technique allows for a completely percutaneous approach under conscious sedation $(11,14,15)$.

A major limitation associated with percutaneous TAxTAVR involves the lack of compressibility of the axillary artery. This can often be dealt with by compressing the vessel against the thoracic cage. However, if the puncture is deep, it is problematic and can be difficult to obtain hemostasis after removal of the sheath. Although lifethreatening bleeding and major vascular complications are rare, placement of a peripheral stent may be necessary. In 


\begin{tabular}{|c|c|c|c|}
\hline & Axillary & Trans-apical/trans-aortic & $P$ value \\
\hline $\mathrm{N}$ & 1,180 & 1,180 & \\
\hline All-cause 30 -day mortality & $58(5.3 \%)$ & $94(8.4 \%)$ & 0.005 \\
\hline All stroke & $72(6.3 \%)$ & $35(3.1 \%)$ & 0.0002 \\
\hline New-onset atrial fibrillation & $23(2.0 \%)$ & $150(13.0 \%)$ & $<0.0001$ \\
\hline All readmissions & $120(11.6 \%)$ & $157(15.1 \%)$ & 0.03 \\
\hline New requirement for dialysis & $8(0.7 \%)$ & $28(2.5 \%)$ & 0.001 \\
\hline Life-threatening bleeding & $5(0.5 \%)$ & $6(0.6 \%)$ & 0.78 \\
\hline Major vascular complication & $29(2.5 \%)$ & $20(1.7 \%)$ & 0.19 \\
\hline New permanent pacemaker & $133(11.7 \%)$ & $115(10.1 \%)$ & 0.14 \\
\hline Length of index hospital stay (days) & $3.0(2.0-5.0)$ & $6.0(4.0-8.0)$ & $<0.0001$ \\
\hline Length of ICU stay (hrs) & $26.3(19.5-48.0)$ & $47.0(25.0-95.5)$ & $<0.0001$ \\
\hline
\end{tabular}

Source: Dahle TG, Kaneko T, McCabe JM. Outcomes Following Subclavian and Axillary Artery Access for Transcatheter Aortic Valve Replacement. JACC Cardiovasc Interv. 2019. doi:10.1016/j.jcin.2019.01.219.

\begin{tabular}{llll}
\hline \multicolumn{4}{|c}{ Table 3 Clinical outcomes for the matched subclavian/axillary access and trans-femoral access groups using the CoreValve } \\
\hline N & Subclavian/axillary & Transfemoral & P value \\
\hline Age & 202 & 202 & $80.2 \pm 9.7$ \\
\hline STS PROM (\%) & $80.8 \pm 8.1$ & $9.8 \pm 5.5$ & 0.506 \\
\hline All-cause 30-day mortality & $9.7 \pm 5.9$ & $12(5.9 \%)$ & 0.845 \\
\hline All-cause 1-year mortality & $11(4.9 \%)$ & $50(24.8 \%)$ & 0.833 \\
\hline Any stroke or transient ischemic attack & $47(23.3 \%)$ & $10(5.0 \%)$ & 0.703 \\
\hline Major bleeding & $15(7.5 \%)$ & $46(22.8 \%)$ & 0.295 \\
\hline Major vascular complication & $56(27.8 \%)$ & $21(10.4 \%)$ & 0.259 \\
Acute kidney injury & $24(11.9 \%)$ & $20(10.0 \%)$ & 0.639 \\
\hline New permanent pacemaker & $29(14.4)$ & $53(26.4 \%)$ & 0.180 \\
\hline $\begin{array}{l}\text { Source: Popma JJ, Michael Deeb G, Yakubov SJ, et al. Transcatheter aortic-valve replacement with a self-expanding valve in low-risk } \\
\text { patients. N Engl J Med. 2019. doi: 10.1056/NEJMoa1816885. }\end{array}$ &
\end{tabular}

a worst-case scenario, conversion to an open procedure may become necessary. It is vital to maintain wire access in case balloon tamponade is needed. Hematomas at the percutaneous access site are not uncommon and can occur immediately or within a few days after TAVR. In general, they can be managed conservatively and typically resolve spontaneously over time.
Another major disadvantage is the high reported risk of stroke (6). The TVT registry reported that low-volume centers might have higher complication rates (6). With operator experience however, the risk may become lower (6). The use of the Sentinel device may decrease the incidence of stroke, but more data is necessary to draw a conclusion. Other alternative access sites such as trans-carotid and 


\begin{tabular}{|c|c|c|c|}
\hline & Trans-axillary TAVR & Trans-femoral TAVR & $P$ value \\
\hline Age & $78.53 \pm 2.68$ & $80.8 \pm 8.1$ & 0.16 \\
\hline STS Score (\%) & $13.8 \pm 10.18$ & $12.45 \pm 9.26$ & 0.41 \\
\hline Major vascular complications (\%) & $40 / 618(6.5 \%)$ & 198/3,886 (5.1\%) & 0.36 \\
\hline Life-threatening bleeding (\%) & $34 / 343(9.9 \%)$ & $29 / 343(8.4 \%)$ & 0.75 \\
\hline Major bleeding & $54 / 369(14.6 \%)$ & $79 / 3,270(2.4 \%)$ & 0.35 \\
\hline
\end{tabular}

trans-caval approaches have shown promising results and may overcome the disadvantages of TAx-TAVR $(16,17)$.

Patients with a past medical history of coronary artery bypass graft and a patent LIMA to left anterior descending artery bypass graft present a technical challenge that must be taken into consideration before deciding to proceed with TAxTAVR (18). Our experience has been that if the diameter at the LIMA takeoff is larger than the minimal required diameter for the specific device, the incidence of myocardial ischemia is extremely rare. Nonetheless, electrocardiogram monitoring should be used during the advancement of the large-bore sheath down the subclavian artery to ensure adequate perfusion to the territory perfused by the LIMA (18). Systolic blood pressure should also be kept higher, ideally greater than $100 \mathrm{mmHg}$, to maximize perfusion of the LIMA (18).

\section{Conclusions}

Advancements in alternative access techniques, TAVR technologies, and preference for a less invasive approach have allowed the application of TAx-TAVR to grow rapidly among patients with prohibitive femoral access. Surgeon familiarity with the axillary artery and the possibility of a percutaneous approach has made this procedure more popular. The reported outcomes have shown this to be a safe procedure, though further efforts to decrease the rate of stroke are needed.

\section{Acknowledgments}

All sketches and drafts are the copyright of the artist Beth Croce with license for exclusive use in Annals of Cardiothoracic Surgery. Contact beth@bioperspective.com for details about license for additional use by authors.

\section{Footnote}

Conflicts of Interest: TK, MD is a speaker for Edwards LifeSciences (Irvine, California), Abbott Laboratories (Lake Bluff, Illinois), and Medtronic (Minneapolis, Minnesota); a consultant for Baylis Medical and 4C Medical. Dr. PBS is a speaker for Edwards Lifesciences. The other authors have no conflicts of interest to declare.

Open Access Statement: This is an Open Access article distributed in accordance with the Creative Commons Attribution-NonCommercial-NoDerivs 4.0 International License (CC BY-NC-ND 4.0), which permits the noncommercial replication and distribution of the article with the strict proviso that no changes or edits are made and the original work is properly cited (including links to both the formal publication through the relevant DOI and the license). See: https://creativecommons.org/licenses/by-nc-nd/4.0/.

\section{References}

1. Leon MB, Smith CR, Mack M, et al. Transcatheter aortic- 
valve implantation for aortic stenosis in patients who cannot undergo surgery. N Engl J Med 2010;363:1597-607.

2. Mack MJ, Leon MB, Thourani VH, et al. Transcatheter Aortic-Valve Replacement with a Balloon-Expandable Valve in Low-Risk Patients. N Engl J Med 2019;380:1695-705.

3. Popma JJ, Deeb GM, Yakubov SJ, et al. Transcatheter Aortic-Valve Replacement with a Self-Expanding Valve in Low-Risk Patients. N Engl J Med 2019;380:1706-15.

4. FDA. FDA expands indication for several transcatheter heart valves to patients at low risk for death or major complications associated with open-heart surgery. FDA News Release 2019. Available online: https://www.fda. gov/news-events/press-announcements/fda-expandsindication-several-transcatheter-heart-valves-patients-lowrisk-death-or-major

5. Bavaria JE. TAVR Update: New Insights and Perspectives from the U. S. National STS/ACC TVT Registry 2020.

6. Dahle TG, Kaneko T, McCabe JM. Outcomes Following Subclavian and Axillary Artery Access for Transcatheter Aortic Valve Replacement: Society of the Thoracic Surgeons/American College of Cardiology TVT Registry Report. JACC Cardiovasc Interv 2019;12:662-9.

7. Gleason TG, Schindler JT, Hagberg RC, et al. Subclavian/ Axillary Access for Self-Expanding Transcatheter Aortic Valve Replacement Renders Equivalent Outcomes as Transfemoral. Ann Thorac Surg 2018;105:477-83.

8. Blackstone EH, Suri RM, Rajeswaran J, et al. Propensitymatched comparisons of clinical outcomes after transapical or transfemoral transcatheter aortic valve replacement: a placement of aortic transcatheter valves (PARTNER)-I trial substudy. Circulation 2015;131:1989-2000.

9. O'Hair DP, Bajwa TK, Popma JJ, et al. Direct Aortic Access for Transcatheter Aortic Valve Replacement Using a Self-Expanding Device. Ann Thorac Surg

Cite this article as: Harloff MT, Percy ED, Hirji SA, Yazdchi F, Shim H, Chowdhury M, Malarczyk AA, Sobieszczyk PS, Sabe AA, Shah PB, Kaneko T. A step-by-step guide to trans-axillary transcatheter aortic valve replacement. Ann Cardiothorac Surg 2020;9(6):510-521. doi: 10.21037/acs-2020-av-79
2018;105:484-90.

10. Rogers T, Lederman RJ. Percutaneous transaxillary access for TAVR: Another opportunity to stay out of the chest. Catheter Cardiovasc Interv 2018;91:157-8.

11. Bapat V, Tang GHL. Axillary/Subclavian Transcatheter Aortic Valve Replacement: The Default Alternative Access? JACC Cardiovasc Interv 2019;12:670-2.

12. Blanke P, Naoum C, Dvir D, et al. Predicting LVOT Obstruction in Transcatheter Mitral Valve Implantation: Concept of the Neo-LVOT. JACC Cardiovasc Imaging 2017;10:482-5.

13. Amat-Santos IJ, Rojas P, Gutiérrez H, et al. Transubclavian approach: A competitive access for transcatheter aortic valve implantation as compared to transfemoral. Catheter Cardiovasc Interv 2018;92:935-44.

14. Svensson LG. Percutaneous Treatment of Aortic Valve Disease. In: Cohn LH. editor. Cardiac Surgery in the Adult. 4th edition. McGraw-Hill Education. 2012:783-9.

15. Petronio AS, De Carlo M, Giannini C, et al. Subclavian TAVI: more than an alternative access route. EuroIntervention 2013;9 Suppl:S33-7.

16. Lederman RJ, Babaliaros VC, Rogers T, et al. The Fate of Transcaval Access Tracts: 12-Month Results of the Prospective NHLBI Transcaval Transcatheter Aortic Valve Replacement Study. JACC Cardiovasc Interv 2019;12:448-56.

17. Overtchouk P, Folliguet T, Pinaud F, et al. Transcarotid Approach for Transcatheter Aortic Valve Replacement With the Sapien 3 Prosthesis: A Multicenter French Registry. JACC Cardiovasc Interv 2019;12:413-9.

18. Modine T, Sudre A, Collet F, et al. Transcutaneous aortic valve implantation using the axillary/subclavian access with patent left internal thoracic artery to left anterior descending artery: Feasibility and early clinical outcomes. J Thorac Cardiovasc Surg 2012;144:1416-20. 